\title{
Correction to: Sepsis increases perioperative metastases in a murine model
}

Lee-Hwa Tai ${ }^{1}$, Abhirami A. Ananth ${ }^{1,2}$, Rashmi Seth ${ }^{3}$, Almohanad Alkayyal ${ }^{1,2,4}$, Jiqing Zhang 2,5,6, Christiano Tanese de Souza ${ }^{2}$, Phillip Staibano ${ }^{2}$, Michael A. Kennedy ${ }^{2}$ and Rebecca C. Auer ${ }^{1,2,3,7^{*}}$

\section{Correction}

It has been highlighted that the original manuscript [1] contains a typesetting error in Fig. 1 and the Fig. 1c panel has been inadvertently duplicated in panel Fig. 1d. This does not affect the results and conclusions of the article. The correct version of Fig. 1 is included with this Correction. The original article has been updated.

\begin{abstract}
Author details
'Deparment of Biochemistry, Microbiology, and Immunology, Faculty of Medicine, University of Ottawa, Ottawa, Canada. ${ }^{2}$ Center for Innovative Cancer Research, Ottawa Hospital Research Institute, Ottawa, Canada. ${ }^{3}$ Department of Surgery, Division of General Surgery, University of Ottawa, Ottawa, Canada. ${ }^{4}$ Department of Medical Laboratory Technology, University of Tabuk, Tabuk, Saudi Arabia. ${ }^{5}$ Department of Neurosurgery, The Second Hospital of Shandong University, Shandong, China. ${ }^{6}$ Department of Cellular and Molecular Medicine, Faculty of Medicine, University of Ottawa, Ottawa, Canada. ${ }^{7}$ Ottawa General Hospital, 501 Smyth Road, 1617 CCW, Box 134,

Ottawa, ON K1H8L6, Canada.
\end{abstract}

Received: 19 March 2018 Accepted: 19 March 2018

Published online: 17 April 2018

\section{Reference}

1. Tai $L H$, et al. Sepsis increases perioperative metastases in a murine model. BMC Cancer. 2018;18:277. https://doi.org/10.1186/s12885-018-4173-4.

\footnotetext{
* Correspondence: rauer@ohri.ca

'Deparment of Biochemistry, Microbiology, and Immunology, Faculty of Medicine, University of Ottawa, Ottawa, Canada

${ }^{2}$ Center for Innovative Cancer Research, Ottawa Hospital Research Institute, Ottawa, Canada
} 

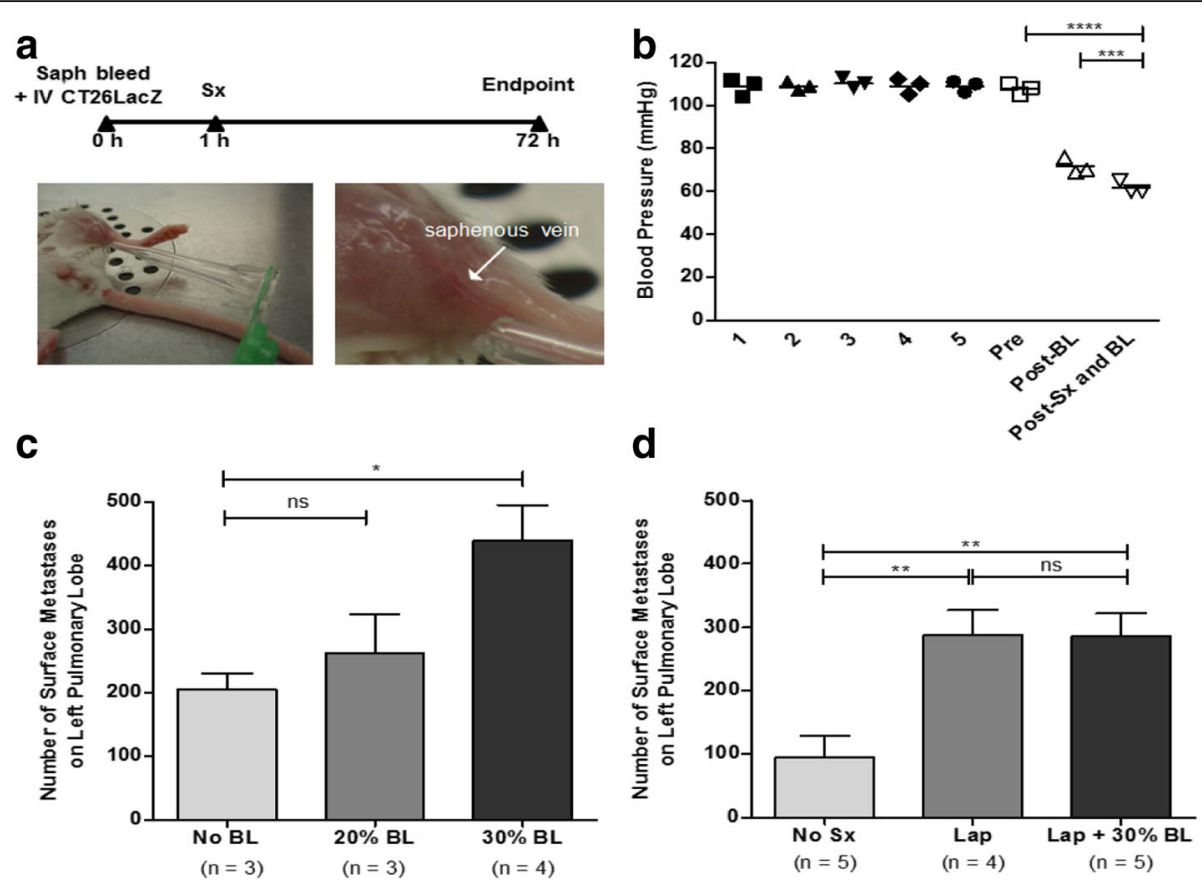

Fig. 1 Hemorrhagic shock does not increase metastatic disease. a Experimental overview. BALB/c mice were bled through the saphenous vein (indicated by the white arrow) and subsequently injected intravenously (IV) through the tail vein with $3 \times 10^{5} \mathrm{CT} 26$ LacZ cells. Approximately $1 \mathrm{~h}$ later, surgical stress (sx) was generated by laparotomy (Lap) $(5 \mathrm{~cm}$ incision). Mice were sacrificed at $72 \mathrm{~h}$ to quantify lung metastases. b Blood pressure is reduced following surgical stress and blood loss. Blood pressure ( $\mathrm{mmHg}$ ) was measured following a 5-day training period (Day 1-5), prior to bleeding (Pre), immediately following bleeding (Post-BL), and immediately following surgical stress (Post-Sx and BL, $n=3$ ). $\mathbf{c}$ Blood loss increases metastatic burden. Lung metastases were measured on Day 3 following no blood loss (no BL, $n=3$ ) or 20\% (20\% BL, $n=3$ ) or $30 \%$ blood loss ( $30 \% \mathrm{BL}, n=4)$. $\mathbf{d}$ Blood loss does not increase metastatic disease in conjunction with surgical stress. Lung metastases were measure on Day 3 in mice that did not undergo surgical stress (No Sx, $n=5)$ and animals undergoing a laparotomy (Lap, $n=4)$ alone or in combination with $30 \%$ blood loss (Lap $+30 \% \mathrm{BL}, n=5)$. Error bars represent \pm SEM 